\title{
The Effect of a Culturally Tailored Web-based Physical Activity Promotion Program on Asian American Midlife Women's Depressive Symptoms
}

Asian/Pacific Island Nursing Journal Volume 1(4): 162-173

(C)Author(s) 2016

http://digitalscholarship.unlv.edu/apin/

\author{
Wonshik Chee ${ }^{a}$, Sangmi Kim ${ }^{b}$, Xiaopeng Ji ${ }^{b}$, Sooyoung Park ${ }^{b}$, Eunice Chee ${ }^{\mathrm{c}}$, Hsiu-Min \\ Tsaid, and Eun-Ok Im ${ }^{\mathrm{a}}$
}

\begin{abstract}
The benefits of physical activities on depressive symptoms have increasingly been reported in the literature, but the effect through which a Web-based physical activity promotion program alleviates depressive symptoms is not clearly known, especially among ethnic minority midlife women. The purpose of this pilot randomized control study is to examine the preliminary efficacy of the Web-based physical activity promotion program in enhancing the depressive symptoms of Asian American midlife women through increasing physical activity. This study adopted a randomized repeated measures pretest/posttest control group design. This study consisted of two groups of research participants: 18 in an intervention group and 15 in a control group. By using multiple instruments, the participants' background and health status, depressive symptom experience, and physical activity experience were measured at three time points (pre-, post 1-month, and post 3-months). The data were analyzed using a modified intent-to-treat linear mixed-model growth curve analysis. After controlling for covariates, random intercept, and random slope, only discrimination stress showed statistical significances in the group effect $(0.18, p=.08$ for control) and time effect $(-0.04, p=.04)$, but not in the group $\times$ time effect $(p=.51)$. The active living habits scores showed statistical significances in the group effect $(0.82, p<0.01$ for control), time effect $(0.29, p<0.01)$, and group $\times$ time effect $(-0.31, p=0.03$ for control). Findings support the significant effect of the Web-based physical activity promotion program on the women's discrimination stress and active living habits.
\end{abstract}

Keywords: web-based intervention, depression, physical activity, midlife women, Asian American

With an increasing number of aging racial/ethnic minorities in the United States (Institute of Medicine, 2008), depression has become an important issue in midlife racial/ethnic minorities. Racial/ethnic minorities often tend not to recognize and/or treat depression (Lagomasino et al., 2005; Sin, Jordan, \& Park, 2011). Furthermore, racial/ethnic minority immigrants are less likely to be diagnosed compared with non-immigrants, even with those from the same race/ethnicity (Gwynn et al., 2008). Immigrants hardly seek treatment or management of their depression and prematurely quit treatment or management of their depression (Lagomasino et al., 2005; Sin et al., 2011). Furthermore, midlife with hormonal changes is the stage of life with various

\footnotetext{
${ }^{a}$ Duke University, USA

${ }^{b}$ University of Pennsylvania

${ }^{c}$ University of North Carolina, Chapel Hill, USA

Corresponding Author:

Wonshik Chee, PhD, Associate Professor

School of Nursing

Duke University

Email: wonshik.chee@duke.edu
}

${ }^{d}$ Chang Gung University of Science and Technology, Taiwan

c) 9 - Creative Commons CC-BY-NC-ND: This article is distributed under the terms of the Creative Commons Attribution 4.0 License (http://creativecommons.org/licenses/by/4.0/) which allows others to download your works and share them with others as long as they credit you, but they can't change them in any way or use them commercially. 
changes that frequently make ethnic minority immigrant women at increased risk of depression (Judd, Hickey, \& Bryant, 2012; Parry, 2010).

Depression has various modes of preventive and therapeutic interventions including pharmacotherapy, psychotherapy, and lifestyle or behavioral modification (Hamer, Endrighi, \& Poole, 2012). However, pharmacotherapy is effective only in about $30 \%$ of patients, and some have a partial response to interventions, which necessitates other forms of interventions (Hamer et al., 2012; Trivedi et al., 2006). Many ethnic minority immigrants including Asian American midlife women reportedly prefer lifestyle management interventions including physical activities to other types of interventions mainly due to the misconceptions and stigma attached to mental illness (Gomez, Kelsey, Glaser, Lee, \& Sidney, 2004; Hovey, 2000; Jimenez, Alegría, Chen, Chan, \& Laderman, 2010; Turner \& Avison, 2003). Indeed, lifestyle management strategies including physical activity promotion are widely used as preventive and therapeutic interventions for depression (Gomez et al., 2004; Hovey, 2000; Jimenez et al., 2010; Turner \& Avison, 2003). Specifically, the benefits of physical activities on depressive symptoms have increasingly been reported in the literature (Gomez et al., 2004; Hovey, 2000; Jimenez et al., 2010; Turner \& Avison, 2003).

Despite an increasing number of Web-based physical activity promotion programs, the effect through which a Web-based physical activity promotion program improves depressive symptoms is not clearly known, especially among ethnic minority midlife women (Torres, Sampselle, Ronis, Neighbors, \& Gretebeck, 2013; Wise, Adams-Campbell, Palmer, \& Rosenberg, 2006). Based on previous studies (Im, Chang, Ko, et al., 2012; Im, Ko, et al., 2012; Im, Chang, Chee, \& Chee, 2012), the research team developed the first theory-driven Web-based physical activity promotion program that is culturally tailored for Asian American midlife women by incorporating their unique cultural attitudes toward physical activity. Bandura's Theory of Behavioral Change (Bandura, 1977) guided the development of the program. Attitudes, self-efficacy, and barriers related to physical activity were targeted in the program (see below for more information on the program).

The purpose of this pilot randomized control study was to examine the preliminary efficacy of the Web-based physical activity promotion program (WPAPP) in enhancing depressive symptoms of Asian American midlife women through increasing their physical activity. The hypotheses that were tested included:

- Hypothesis 1. The intervention group would show significantly greater improvements than the control group in self-reported depressive symptom experience (operationalized into depressive symptom scores, self-reported stress [acculturation stress, life stress, and discrimination stress], and perceived social support [perceived isolation and personal resource]) from a pre-test to the two follow-up time points (post 1 month [Time 1] and post 3 months [Time 2]). In this paper, these outcome variables measuring self-reported depressive symptom experience are called the outcome variables related to depressive symptoms.

- Hypothesis 2. The intervention group would show significantly greater improvements than the control group in self-reported physical activity experience (operationalized into self-reported physical activity scores, attitudes toward physical activity, self-efficacy, perceived barriers, and social influences) from pre-test to Times $1 \& 2$. In this paper, these outcome variables measuring self-reported physical activity experience are called the outcome variables related to physical activity.

\section{Method}

This study adopted a randomized repeated measures pretest/posttest (pre-test, post 1-month, \& post 3-months) control group design. The study included 33 participants: (a) 18 Asian American midlife women who used the WPAPP and the website on physical activity guidelines by the Centers for Disease Control and Prevention (CDC), and (b) 15 Asian American midlife women who used only the CDC website. Among various sub-ethnic groups of Asian Americans, only Chinese and Korean American midlife women were targeted in this pilot study because the WPAPP was developed using three languages including English, Mandarin Chinese, and Korean. Also, Chinese Americans were chosen because they are the largest sub-ethnic group within Asian Americans (U.S. Census Bureau, 2011). Korean Americans were chosen because their depression rate was the highest among Asian Americans (Park \& Bernstein, 2008). The control group was asked to maintain their usual information searches through existing resources. The CDC website provided basic information on physical activity (health benefits, aerobic exercise, musclestrengthening activities, tips on getting active, and several successful stories). However, they did not provide any component of the WPAPP (e.g., individual and/or group coaching/support, ethnic-specific educational modules or online resources, etc.). The study was conducted from January 2014 to May 2016 under the Institutional Review Board approval of the institute where the authors worked. 


\section{Samples and Settings}

A total of 33 Asian American (Chinese or Korean) midlife women were nationally recruited to an Intervention Group (IG) and a Control Group (CG). The settings were Internet communities or groups for Chinese or Korean Americans in the United States. The literature indicated that nationally recruiting Asian American midlife women through Internet communities or groups was feasible and highly effective (McDonnell, Lee, Kazinets, \& Moskowitz, 2010). The participants were self-reported Chinese or Korean American midlife women aged 40 to 60 years whose parents and grandparents were of Chinese or Korean descent; who could read and write English, Mandarin Chinese, or Korean; who currently resided in the United States; who were online through computers or mobile devices (smartphones and tablets); and who had experienced depression during the past two weeks (scored at least 1 on the Patient Health Questionnaire, which was equivalent to the cut-point of minimal depressive symptoms; Spitzer, Kroenke, Williams, \& the Patient Health Questionnaire Primary Care Study Group, 1999). Internet searches were conducted using Google, Yahoo!, and Bing, and the Internet communities/groups for Chinese or Koreans in the United States. were identified/verified. Then, the administrators of the first 10 Internet communities on the verified lists were asked to post the study announcement, and additional 10 Internet communities were weekly contacted for the study announcement until the target number of participants was recruited. When potential participants visited the project website and clicked "I am interested in participating in the study" after viewing the informed consent, they were screened to check if they met the inclusion and exclusion criteria and randomized into IG and CG using an automated random number generator accessible through the website. The sample size required to fully test the hypotheses of this study was larger than the recruited sample size. Yet, 33 is an adequate sample size for a pilot intervention study (Polit \& Beck, 2014). More detailed information on issues in the participant recruitment of the study can be found elsewhere (Chee et al., 2016).

\section{The Web-based Physical Activity Promotion Program (WPAPP)}

As mentioned above, WPAPP was theoretically based on Bandura's Theory of Behavioral Change. Most behavior change programs based on Bandura's theory include coaching/support and information as part of the program content (AHRQ, 2014). Likewise, to increase physical activity, WPAPP targeted changing midlife Chinese and Korean Americans' physical activity behavior through providing individual and group coaching/support and information. At the same time, WPAPP provided educational modules and online forums on midlife women's depressive symptoms and physical activity. Then, individual and group coaching/support reportedly changes physical activity behaviors through enhancing attitudes, self-efficacy, perceived barriers, and social influences related to physical activity Information is also reported to change the women's attitudes, self-efficacy, and perceived barriers (Baumeister \& Finkel, 2010; Lee, Arthur, \& Avis, 2008; Rydell et al., 2005). Then, the increase in physical activity through WPAPP was subsequently expected to improve depressive symptoms of Chinese and Korean American midlife women.

WPAPP was designed to include three components in three languages (English, Mandarin Chinese, and Korean): (a) a social media site with chat functionality, (b) interactive online educational sessions, and (c) online resources. The social media site allowed the participants to share their own experiences and to receive individual and group coaching/support using Fitbits from culturally matched peers and health care providers. Interactive online educational sessions were available in English, Mandarin Chinese, and Korean. The modules included (a) benefits of physical activity within cultural contexts; (b) impacts of physical activity on various health conditions within cultural contexts; (c) types of physical activity (general and culture-specific); (d) tips to increase physical activity in busy daily schedule within cultural contexts; (e) general and culture-specific physical activity guidelines; (f) aerobic and muscle-strengthening activities within cultural contexts; $(\mathrm{g})$ the guidelines for physical activity in inactive, active, and highly active adults within cultural contexts; (h) flexibility activities, warm-up and cool-down within cultural contexts; (i) a weight control plan and a culturally matched case study; (j) achieving target levels of physical activity within cultural contexts; $(\mathrm{k})$ various short videos on physical activity (general and culture-specific); (l) office exercises (general and culture specific); and (m) examples of general and culture-specific exercises that could be easily done with families without any necessary equipment. Online resources included 20 links to resources in English, Mandarin Chinese, or Korean from scientific authorities including the National Institutes of Health, American Diabetic Association, Korean Centers for Disease Control and Prevention, among others. To ensure cultural competence, WPAPP was reviewed by five experts against the criteria for rigor in cross-cultural research (Aegisdóttir, Gerstein, \& Çinarbaş, 2008). Then, the educational modules and online resources were supplemented with additional content, and the structure of WPAPP was revised to be more user-friendly. 


\section{Instruments}

\section{Questions on Background Characteristics and Disease Status}

Multiple questions were used for the study to measure background characteristics and disease status of the participants, which served as covariates. The questions included those on age, ethnicity, education, employment, degree of difficulty in paying for basics including food, housing, clothing, and health care (socioeconomic status [SES] proxy), and diagnosed disease(s). Ethnicity was classified into "Chinese" and "Korean." Education was categorized into "no school," "elementary school/graduated," "middle school/graduated," "high school/graduated," "partial college," "college/graduated," and "graduate degree." Employment was categorized into "employed" and "not employed." SES was determined using self-reports on the degree of difficulty in paying for basics, and classified to "very hard to pay for basics (low)," "somewhat hard to pay for basics (middle)," and "not hard to pay for basics (high)." Also, existing diagnosed diseases (yes or no) were asked to assess the women's disease status.

To measure acculturation, eight questions on the country of birth, age at immigration, years of stay in the United States, and degree of acculturation were used. First, the participants were asked their country of birth. Then, if they were not born in the United States, their age at immigration and years of stay in the United States were measured. The degree of acculturation was measured using five Likert-scale questions on foods, music, customs, language, and close friends (from 1 [exclusively Korean] to 5 [exclusively American]) from the Suinn-Lew Asian Self-identity Acculturation scale (SL-ASIA; Suinn, Ahuna, \& Khoom, 1992). The participants' disease status was measured using two questions on weight and height (used to calculate the Body Mass Index; five questions on perceived general health, self-reported diagnosed clinical depression (major depression and dysthymia), depression medication, other diagnosed diseases and medication, and access to health care; seven questions to determine menopausal status; two questions on family history of depression only among first-degree relatives (yes or no) and among other relatives (yes or no); and two questions on family history of other psychiatric diseases only among first-degree relatives (yes or no) and among other relatives (yes or no). These questions were used without any issues/concerns in previous studies (Im, Chang, Ko, et al., 2012; Im, Ko, Chee, \& Chee, 2015; Im, Shin, \& Chee, 2008).

\section{The Center for Epidemiologic Studies De- pression Scale-Korean}

The Center for Epidemiologic Studies Depression Scale (Noh, Avison, \& Kaspar, 1992) was used to measure the frequency of depressive symptoms in the last week. The Center for Epidemiologic Studies Depression Scale includes 20 items on the level of depression, which are usually summed to depression scores. The possible range of scores were 0 60 (larger scores mean higher depression). The cutoff score to determine the presence of depressive symptoms is 21 (Noh et al., 1992). The usual cutoff score is 16 , but 21 was selected for this study while following the recommendation by previous studies among Asian Americans (Jang, Kim, \& Chiriboga, 2005).

\section{Acculturation Stress Scale}

The Acculturation Stress Scale (de Snyder, 1987) includes 12 questions to measure the level of stress due to language difficulties, economic and social conflicts, cultural value, and role conflict that are from the acculturation process related to immigration transition. Individual items use a 4-point Likert scale $(0=$ not stressful to $3=$ very stressful $)$. Content and construct validity was supported, and Cronbach's alpha was.73. in previous studies (Shin, Han, \& Kim, 2007).

\section{Social Readjustment Rating Scale}

The Social Readjustment Rating Scale (Masuda \& Holmes, 1978) includes 57 items on life events (e.g., empty nest, health changes of family member, living condition changes, etc.), and each item has different weighting indicating the severity of the stressor (ranging from 0 to 100). In this study, the Social Readjustment Rating Scale scores were calculated by summing all items. Concurrent validity was supported and Cronbach's alpha was .62 to .93 in previous studies (Lavallee, \& Flint, 1996; Masuda \& Holmes, 1978).

\section{Discrimination Scale}

The Discrimination Scale (Essed, 1991) includes nine items on self-reported frequencies of discrimination experience. Each item uses a 6-point Likert scale $(0=$ never to $5=$ almost every day $)$. The Discrimination Scale also includes one additional question on perceived reasons for discrimination with multiple choices (Bernstein, Park, Shin, Cho, \& Park, 2011). Construct and content validity were supported, and Cronbach's alpha was .90 to .91 in previous studies (Bernstein et al., 2011). 


\section{Perceived Isolation Scale}

The Perceived Isolation Scale measures perceived interactions (social isolation) and includes six items of perceived social support and three items on interactions/loneliness (3-point Likert scales) with 2 additional questions on interactions with peers and moderator(s). In this study, all the items were summed to calculate the interaction scores. Cronbach's alpha was .86 , and content validity was supported in previous studies (Weissman, Leaf, Holzer, Myers, \& Tischler, 1984).

\section{Personal Resource Questionnaire}

The shortened version of Personal Resource Questionnaire (Weinert, 2003) includes 25 items and 15 items of them measure respondents' perceived level of social support. The Personal Resource Questionnaire has 7-point Likert scale items in five dimensions. The dimensions include "provision for attachment/intimacy," "social integration," "opportunity for nurturing behavior," "reassurance of worth," and "the availability of informational, emotional, and material helps." Construct validity was supported; test-retest reliability was .72 and Cronbach's alpha was .85 to .93 in previous studies (Han, Kim, \& Weinert, 2002; Shin et al., 2007; Weinert, 2003).

\section{Kaiser Physical Activity Survey (KPAS)}

The KPAS (Ainsworth, Sternfeld, Richardson, \& Jackson, 2000) includes four subscales: household/caring (11 items), occupational (12 items), active living habits (4 items), and sports/exercise (15 items). Individual items use a 5-point scale. In this study, the items with negative wordings were reverse coded, and the items in each subscale were averaged $(1<$ the mean score per subscale $<5)$. Then, all items were summed to the KPAS scores. In previous studies, the KPAS scores were found to be significantly correlated with the Caltrac accelerometer measured physical activity, physical activity records, and cardiorespiratory fitness (VO2 peak; Ainsworth et al., 2000). Test-retest correlation was .79 to .91; Cronbach's alpha was .84, and construct and content validity was supported in previous studies (Im et al., 2014; Im, Chee, Lim, Liu, \& Kim, 2008).

Questions on Attitudes Toward Physical Activity, Subjective Norm, Perceived Behavioral Control, and Behavioral Intention

The Questions on Attitudes Toward Physical Activity, Subjective Norm, Perceived Behavioral Control, and Behavioral Intention was directly developed based on the theory of planned behavior by Armitage (Armitage, 2005), and has four subscales: (a) attitudes, (b) social influences (subjective norm), (c) perceived behavioral control, and (d) behavioral intention. Only the attitude and social influences subscales were used; the attitude subscale included six bipolar $(-3$ to +3$)$ semantic differential scale items and the social influences subscale included three unipolar $(+1$ to +7$)$ scale items. In this study, the attitude and social influences scores were calculated by adding the items in each subscale. Cronbach's alpha was .87 to .90 , and content validity was supported in a previous study (Armitage, 2005).

\section{Physical Activity Assessment Inventory}

The Physical Activity Assessment Inventory (Haas, 2001) includes 13 items that ask the participants to rate their confidence to perform usual physical activity in various circumstances. The responses could range from 0 (cannot do at all) to 100 (certainly can do). Cronbach's alpha was .95 , and content validity was supported in previous studies (Im, Chee, et al., 2008; Im et al., 2010; Im, Chang, Ko, et al., 2012).

\section{Modified Barriers to Health Activities Scale}

The modified Barriers to Health Activities Scale (Stuifbergen \& Becker, 1994) includes 16 items on barriers to regular physical activity that ask the participants to rate the frequency of interferences with their regular physical activity due to the problem listed in individual items (on a 4-point scale from $1=$ never to 4 = routinely). All items were summed to the Barriers to Health Activities Scale scores, which represented the frequency of occurrence of self-perceived barriers. Cronbach's alpha was .87 to .88 , and content and construct validity was supported in previous studies (Im, Chee, et al., 2008; Im et al., 2010; Im, Chang, Ko, et al., 2012).

\section{Data Collection Procedures}

When a potential participant accessed the project website after reviewing the study announcement, she was asked to choose a language version and click to enter the "informed consent" in her chosen language. By clicking the "I agree to participate" button, she gave her consent. After checking that she met the inclusion and exclusion criteria, she was automatically assigned a serial number and randomized into one of the two groups (IG and $\mathrm{CG}$ ) using an automated random number generator accessible through the website. She was then automatically linked to the questionnaire to complete (pre-test). When this was completed, she was provided with IDs and passwords that were randomly assigned by the researchers; whenever she visited the website, she needed to use the IDs and passwords to log in the site. If she wanted, she was able to change her 
identifications (IDs) and passwords at her convenience. Then, both groups were provided with an electronic instruction sheet on when they needed to come back (weekly) and fill out additional questionnaires (post 1month and post 3-months) and/or use the program (weekly). Also, both groups were provided with the CDC website address through the project website and asked to use the website during the study period. The control group was asked to maintain their usual information searches through existing resources. Every two weeks, the participants received reminders and thank you emails. Two weeks before the end of the 1st month and the 3rd month, both groups were contacted and asked to fill out the next set of the instruments by the end of the 1 st and $3 \mathrm{rd}$ month.

\section{Data Analysis}

The data were automatically saved in ASCII format. ID numbers were randomly assigned and attached to the data. ASCII files containing the encrypted data were copied to a removable hard disk back-up system and kept in a locked file cabinet. SAS version 9.4 (SAS Institute, Cary, NC) was used for all analyses. The variables were described using descriptive statistics including frequencies, percentages, means, and standard deviations. All outcome variables were examined for normality. Non-normal variables (e.g., Acculturation Stress Scale, Social Readjustment Rating Scale, and Discrimination Scale scores) were log-transformed before analyses. Given the small sample size, the pre-test values of descriptors and outcomes were examined for differences between Internet communities and groups for Chinese or Koreans using Fisher's exact tests for categorical variables and Wilcoxon rank sum tests for continuous variables. An alpha level of .10 was used to determine the statistical significances. Missing data were imputed by the mean substitution only if missing items were less than $20 \%$. The analyses of outcome data were conducted on a modified intent-to-treat basis: all participants were included in the analyses (except those who did not complete all three times of online surveys).

To test Hypotheses 1 and 2, all outcome variables measured over time were examined using a mixed-model growth curve analysis with both fixed and random effects in the model. The fixed effects of time (pre-test, post 1-month, and post 3-months), treatment arm, and a time $\times$ treatment arm interaction were included in the model. Random intercept and random slope (only with a significant time fixed effect) were modeled to represent the subject level deviation from the pre-test grand mean of and the mean change rate in the outcome variables over time, respectively. A separate mixed model was generated for each outcome. Mixed models with PROC mixed were estimated with the maximum likelihood estimation, unstructured covariance structure, and Kenward-Roger degree of freedom method. This degree of freedom method was considered to be ideal for this study because 1) it could be used in models with unbalanced designs, 2) could handle complex covariance structures, and 3) could particularly adjust for the small sample bias (Bell, Ene, Smiley, \& Schoeneberger, 2013). The pre-specified covariates were age, ethnicity, educational attainment, employment status, household income, and diagnosed diseases. With nested models, the Akaike information criterion (AIC) was utilized to compare the overall model fit and to select the best-fitting pattern (Akaike, 1974).

\section{Results}

\section{Background Characteristics of the Partici- pants}

The background characteristics of study participants at pre-test are summarized in Tables 1 and 2. The average age at enrollment was 41.9 years $(S D=$ 9.6). About $61 \%$ of the participants earned a graduate degree; approximately $70 \%$ were employed, and about $71 \%$ did not report difficulty paying for basics. Also, about $82 \%$ did not have a diagnosed disease. $\mathrm{CG}$ and IG did not significantly differ in most of their background characteristics at pre-test. However, they differed in ethnicity, the active living habits scores, the total physical activity scores, and the social influence scores. More Chinese participants $(77.8 \%)$ were assigned to IG than Korean participants $(22.2 \%)$ while it was opposite among CG (see Table 1). At pre-test, CG had higher active living habits scores and total physical activity scores than IG. However, IG had greater social influences scores than CG (see Table 2).

\section{The Effects on Depressive Symptoms (Hy- pothesis 1)}

Depression did not significantly improve over time for both $\mathrm{CG}$ and IG $(p=0.68)$. After controlling for the covariates, random intercept, and random slope, only discrimination stress showed statistical significances in the group effect $(0.1795, p$ $=.08$ for control $)$ and time effect $(-0.0371, p=.04)$, but not in the group $\times$ time effect $(p=.51)$. CG reported higher discrimination stress than IG at pretest. While discrimination stress for both CG and IG tended to decrease over time, the time slope did not differ among the groups (see Table 3 ). In general, the adjustment of covariates improved a model fit for each of the outcome variables related to depressive symptoms and reduced the extent of between-subject's variances in the pre-test outcomes. 
Chee et al.: Physical Activity Promotion Program

Table 1. Comparison of Characteristics Between Control and Intervention Groups at Pre-test

\begin{tabular}{|c|c|c|c|c|}
\hline Characteristic & $\begin{array}{l}\text { Control } \\
(n=15)\end{array}$ & $\begin{array}{c}\text { Intervention } \\
\quad(n=18)\end{array}$ & $\begin{array}{c}\text { Total } \\
(n=33)\end{array}$ & $P-$ Value \\
\hline Age $(M \pm S D)$ & $41.0 \pm 12.5$ & $42.7 \pm 6.8$ & $41.9 \pm 9.6$ & 0.65 \\
\hline \multicolumn{5}{|l|}{ Ethnicity (\%) } \\
\hline Korean & 53.3 & 22.2 & 36.4 & \multirow[t]{2}{*}{$0.08^{\dagger}$} \\
\hline Chinese & 46.7 & 77.8 & 63.6 & \\
\hline \multicolumn{5}{|l|}{ Education $(\%)$} \\
\hline High school/graduated & 6.7 & 11.1 & 9.1 & \multirow[t]{4}{*}{0.77} \\
\hline Partial college & 6.7 & 5.6 & 6.1 & \\
\hline College/graduated & 33.3 & 16.7 & 24.2 & \\
\hline Graduate degree & 53.3 & 66.7 & 60.6 & \\
\hline \multicolumn{5}{|l|}{ Employment (\%) } \\
\hline No & 33.3 & 27.8 & 30.3 & \multirow[t]{2}{*}{1.00} \\
\hline Yes & 66.7 & 72.2 & 69.7 & \\
\hline \multicolumn{5}{|l|}{ Annual family income $(\%)$} \\
\hline Very hard to pay for basics & 0.0 & 0.0 & 0.0 & \multirow[t]{3}{*}{1.00} \\
\hline Somewhat hard to pay for basics & 26.7 & 27.8 & 27.3 & \\
\hline Not hard to pay for basics & 73.33 & 72.2 & 72.7 & \\
\hline \multicolumn{5}{|l|}{ Diagnosed diseases $(\%)$} \\
\hline No & 86.67 & 77.8 & 81.8 & \multirow[t]{2}{*}{0.66} \\
\hline Yes & 13.3 & 22.2 & 18.2 & \\
\hline
\end{tabular}

Note. ${ }^{a} P$-values were computed by Fisher's exact test for categorical variables and Wilcoxon Rank Sum test for continuous variables. ${ }^{\dagger} p<.10$

Table 2. Comparison of Outcomes Between Control and Intervention Groups at Pre-test

\begin{tabular}{lcccc}
\hline Characteristic $(M \pm S D)$ & $\begin{array}{c}\text { Control } \\
(n=15)\end{array}$ & $\begin{array}{c}\text { Intervention } \\
(n=18)\end{array}$ & $\begin{array}{c}\text { Total } \\
(n=33)\end{array}$ & $P$-Value \\
\hline Depression & $18.4 \pm 12.9$ & $19.4 \pm 9.9$ & $19.0 \pm 11.2$ & 0.62 \\
Acculturation stress & $5.0 \pm 5.5$ & $2.6 \pm 4.9$ & $3.7 \pm 5.3$ & 0.14 \\
Social readjustment stress & $95.4 \pm 105.8$ & $81.2 \pm 86.3$ & $87.7 \pm 94.4$ & 0.55 \\
Discrimination stress & $1.6 \pm 0.8$ & $1.5 \pm 0.5$ & $1.5 \pm 0.6$ & 0.49 \\
Perceived isolation & $-0.1 \pm 3.3$ & $0.2 \pm 2.3$ & $0.1 \pm 2.8$ & 0.60 \\
Personal resource & $73.0 \pm 18.2$ & $70.4 \pm 11.9$ & $71.6 \pm 14.9$ & 0.35 \\
\hline Physical activity & & & \\
$\quad$ Household and family care activities & $2.5 \pm 0.7$ & $2.3 \pm 0.4$ & $2.4 \pm 0.6$ & 0.34 \\
$\quad$ Occupational activities & $2.0 \pm 0.6$ & $2.0 \pm 0.8$ & $2.0 \pm 0.7$ & 0.90 \\
$\quad$ Active living habits & $2.9 \pm 0.7$ & $2.1 \pm 0.9$ & $2.4 \pm 0.9$ & $0.01^{*}$ \\
$\quad$ Participation in sports and exercise & $2.6 \pm 0.7$ & $2.4 \pm 1.1$ & $2.5 \pm 1.0$ & 0.44 \\
$\quad$ Total & $10.0 \pm 1.3$ & $8.8 \pm 1.8$ & $9.3 \pm 1.7$ & $0.08^{\dagger}$ \\
Attitudes toward physical activity & $4.4 \pm 1.1$ & $4.3 \pm 1.4$ & $4.3 \pm 1.3$ & 0.86 \\
Self-efficacy of physical activity & $53.2 \pm 14.6$ & $46.1 \pm 19.3$ & $49.3 \pm 17.5$ & 0.35 \\
Barriers to physical activity & $1.8 \pm 0.4$ & $1.8 \pm 0.4$ & $1.8 \pm 0.4$ & 0.82 \\
Social influence on physical activity & $5.5 \pm 1.3$ & $6.4 \pm 0.6$ & $6.0 \pm 1.0$ & $0.05^{\dagger}$ \\
\hline
\end{tabular}

Note. ${ }^{\text {a }}$-values were computed by Wilcoxon Rank Sum test. ${ }^{\dagger} p<.10,{ }^{*} p<.05,{ }^{* *} p<.01$

\section{The Effects on Physical Activity (Hypothesis 2)}

The total physical activity scores as well as all the subscale scores (except the active living habits scores) did not significantly change over time across the groups. A significant group effect of the outcome variables $(1.34, p=0.02$ for control) indicated higher total physical activity scores in CG compared with IG at pre-test. However, the active living habits scores showed statistical significances in the group effect $(0.82, p=.001$ for control), time effect $(0.29, p<.001)$, and group $\times$ time effect $(-0.23, p=.03$ for control).
Relative to IG, CG had higher active living habits scores at pre-test, but IG experienced much greater improvement in the scores over time compared with CG. Only random intercept, not random slope, was significant in the model.

The physical activity self-efficacy scores exhibited a significant group effect $(11.99, p=.06$ for control) where CG had higher self-efficacy than IG at pre-test (Table 3). Likewise, the covariates incorporated in the models accounted for some of the individual variances in the outcome variables related to physical activity at pre-test. 
Table 3. Comparison of Control and Intervention Groups for Study Outcomes at Pre-test, Post 1 Month, and Post 3 Months

\begin{tabular}{|c|c|c|c|c|c|c|c|}
\hline \multirow[b]{2}{*}{ Group } & \multicolumn{2}{|c|}{ Control $(n=14)$} & \multicolumn{2}{|c|}{ Intervention $(n=13)$} & \multicolumn{3}{|c|}{$P$-Values ${ }^{\text {a }}$} \\
\hline & $M$ & $S D$ & $M$ & $S D$ & Group & Time & Group*Time \\
\hline \multicolumn{8}{|l|}{ Depression } \\
\hline Pre-test & 15.08 & 10.92 & 22.08 & 12.64 & 0.11 & 0.17 & 0.68 \\
\hline Post 1 month & 16.62 & 9.68 & 19.62 & 11.76 & & & \\
\hline Post 3 months & 15.46 & 8.74 & 19.27 & 10.77 & & & \\
\hline \multicolumn{8}{|c|}{ Acculturation stress (transformed) } \\
\hline Pre-test & 1.35 & 1.04 & 0.81 & 1.14 & 0.16 & 0.15 & 0.10 \\
\hline Post 1 month & 1.60 & 0.82 & 1.62 & 1.01 & & & \\
\hline Post 3 months & 1.31 & 0.77 & 1.47 & 0.95 & & & \\
\hline \multicolumn{8}{|c|}{ Social readjustment stress (transformed) } \\
\hline Pre-test & 3.86 & 1.43 & 3.83 & 1.56 & 0.95 & 0.24 & 0.83 \\
\hline Post 1 month & 3.84 & 1.37 & 4.17 & 0.98 & & & \\
\hline Post 3 months & 3.72 & 1.35 & 3.61 & 1.42 & & & \\
\hline \multicolumn{8}{|c|}{ Discrimination stress (transformed) } \\
\hline Pre-test & 0.49 & 0.35 & 0.35 & 0.34 & $0.08^{\dagger}$ & $0.04^{* b}$ & 0.51 \\
\hline Post 1 month & 0.42 & 0.36 & 0.32 & 0.24 & & & \\
\hline Post 3 months & 0.35 & 0.33 & 0.29 & 0.22 & & & \\
\hline \multicolumn{8}{|l|}{ Perceived isolation } \\
\hline Pre-test & -0.59 & 2.49 & 0.36 & 1.85 & 0.74 & 0.76 & 0.16 \\
\hline Post 1 month & 0.15 & 2.35 & 0.52 & 3.97 & & & \\
\hline Post 3 months & -1.19 & 2.84 & 1.36 & 3.00 & & & \\
\hline \multicolumn{8}{|l|}{ Personal resource } \\
\hline Pre-test & 79.00 & 10.66 & 69.48 & 11.70 & 0.24 & 0.92 & 0.84 \\
\hline Post 1 month & 77.46 & 12.41 & 66.00 & 21.52 & & & \\
\hline Post 3 months & 78.15 & 13.46 & 69.76 & 14.68 & & & \\
\hline \multicolumn{8}{|c|}{ Household and family care activities } \\
\hline Pre-test & 2.57 & 0.64 & 2.49 & 0.36 & 0.47 & 0.76 & 0.30 \\
\hline Post 1 month & 2.38 & 0.65 & 2.55 & 0.63 & & & \\
\hline Post 3 months & 2.43 & 0.65 & 2.64 & 0.53 & & & \\
\hline \multicolumn{8}{|l|}{ Occupational activities } \\
\hline Pre-test & 1.77 & 0.57 & 1.98 & 0.84 & 0.70 & 0.18 & 0.16 \\
\hline Post 1 month & 2.15 & 0.56 & 1.93 & 0.56 & & & \\
\hline Post 3 months & 2.14 & 0.60 & 2.04 & 0.59 & & & \\
\hline \multicolumn{8}{|l|}{ Active living habits } \\
\hline Pre-test & 2.90 & 0.58 & 1.88 & 0.81 & $0.00^{* *}$ & $0.00^{* * \mathrm{~b}}$ & $0.03^{*}$ \\
\hline Post 1 month & 2.88 & 0.72 & 2.33 & 0.72 & & & \\
\hline Post 3 months & 3.04 & 0.50 & 2.46 & 0.62 & & & \\
\hline \multicolumn{8}{|c|}{ Participation in sports and exercise } \\
\hline Pre-test & 2.69 & 0.78 & 2.27 & 1.17 & 0.44 & 0.42 & 0.42 \\
\hline Post 1 month & 2.43 & 0.74 & 2.34 & 0.87 & & & \\
\hline Post 3 months & 2.34 & 0.89 & 2.27 & 0.90 & & & \\
\hline Total physical activity & & & & & & & \\
\hline Pre-test & 9.94 & 1.40 & 8.63 & 1.96 & $0.02^{*}$ & 0.23 & 0.41 \\
\hline Post 1 month & 9.68 & 1.27 & 8.82 & 0.92 & & & \\
\hline Post 3 months & 9.94 & 1.45 & 9.25 & 1.32 & & & \\
\hline Attitudes toward physica & & & & & & & \\
\hline Pre-test & 4.39 & 1.08 & 4.29 & 1.49 & 0.87 & 0.17 & 0.76 \\
\hline Post 1 month & 4.39 & 1.20 & 4.13 & 1.21 & & & \\
\hline Post 3 months & 4.56 & 1.22 & 4.56 & 1.05 & & & \\
\hline Self-efficacy of physical & & & & & & & \\
\hline Pre-test & 52.71 & 10.87 & 45.11 & 20.63 & $0.06^{\dagger}$ & 0.34 & 0.17 \\
\hline Post 1 month & 49.07 & 15.47 & 48.42 & 25.70 & & & \\
\hline Post 3 months & 51.67 & 12.47 & 50.38 & 19.42 & & & \\
\hline Barriers to physical acti & & & & & & & \\
\hline Pre-test & 1.73 & 0.35 & 1.88 & 0.44 & 0.59 & 0.63 & 0.70 \\
\hline Post 1 month & 1.71 & 0.39 & 1.80 & 0.41 & & & \\
\hline Post 3 months & 1.75 & 0.51 & 1.96 & 0.71 & & & \\
\hline Social influence on phys & & & & & & & \\
\hline Pre-test & 5.50 & 1.31 & 6.36 & 0.62 & 0.46 & 0.14 & 0.31 \\
\hline Post 1 month & 5.52 & 1.28 & 6.05 & 1.29 & & & \\
\hline Post 3 months & 5.81 & 1.19 & 6.31 & 0.88 & & & \\
\hline
\end{tabular}

Note. ${ }^{\text {a }} \mathrm{P}$-values for Group, Time, and Group*Time are estimated after controlling for age, ethnicity, education, employment, income, diagnosed disease, and random intercept. ${ }^{b}$ For a significant time effect, its random slope was included in the model to examine the improved model fit. ${ }^{\dagger} p<.10,{ }^{*} p<.05,{ }^{* *} p<.01$ 


\section{Discussion}

The findings supported that IG had significantly greater increases than CG in active living habits, but not in other outcome variables related to physical activity (Hypothesis 2). Also, the findings supported that both CG and IG had significant improvements in discrimination stress, but not in other outcome variables related to depressive symptoms (Hypothesis 1).

There could be several possible reasons for the non-significant findings. One of the plausible reasons would be that the intervention period (3-month) of this study might not be long enough to show significant improvement in all domains of physical activity and depressive symptom experience. In most intervention studies on physical activity promotion, significant improvement in physical activity was seen at 6 months and maintained at 12 months compared to controls (Wilbur et al., 2013). Thus, it would be difficult to have significant effects of the 3-month program on physical activity that could subsequently result in significant improvement in depressive symptoms.

Another possible reason would be Asian women's cultural hesitance to report depressive symptoms. In Asian culture, mental illnesses including depression have been stigmatized, and Asians including Chinese and Korean Americans tend not to report depressive symptoms (Spector, 2012). This cultural hesitance might have made the participants underreport their depressive symptoms, which subsequently made it difficult to detect changes in the outcome variables related to depressive symptoms due to small variances. Also, in many Asian cultures, physical activity tends to be broadly defined. For example, Im and Choe (2004) reported that Korean American midlife women broadly defined physical activity and considered only death as physical inactivity. Thus, this broad cultural definition of physical activity (considering any activities as physical activities) might have made the impact of the intervention on self-reported physical activities smaller than the actual difference (due to small variances). Subsequently, a significant change could be noted only in the active living habits scores that might have increased to the greater extent than other physical activity scores.

As mentioned above, one promising finding of the study was the significant findings on improvement in active living habits and discrimination stress. Although the underlying mechanism needs to be further explored, the intervention certainly had a significant impact on at least one aspect of the women's physical activity and one aspect of their depressive symptom experience. Throughout the intervention, WPAPP emphasized a gradual increase in physical activities, which was not necessarily limited to exercise or sports. Particularly, the program encouraged the women to walk more during the day. For example, one of the educational modules suggested various ways to promote physical activities in their daily life schedules (e.g., parking their car as far as possible, taking a walk after lunch at least for $10 \mathrm{~min}$, etc.). In contrast, other types of physical activity (e.g., occupational activities and household and caring activities) usually depend on the women's occupations (e.g., secretary job, housewife) and daily life schedules. Thus, the women might have little control over these types of physical activities. Thus, among the four types of physical activity that were measured in this study, active living habits may be the most appropriate index of the efficacy of WPAPP.

The significant improvement in discrimination stress could be due to the women's participation in the ethnic-specific social media sites with their culturally matched peers and health care providers. Their participation in the social media sites might have empowered them to relieve their daily discrimination stress during the course of the intervention. Social media sites including Facebook are now widely used to share health-related messages and encourage discussions on health topics among their users (Abramson, Keefe, \& Chou, 2015). Despite the relatively recent presence of social media sites, an increasing number of studies began to report the effectiveness of social media functions in enhancing health, especially psychological health, of underserved populations including ethnic minorities (Abramson et al., 2015; Hale, Troxel, Kravitz, Hall, \& Matthews, 2014).

This study includes several limitations that need to be considered in interpreting the study findings. First of all, the small sample size could be a limitation. Second, all the outcome variables were measured based on self-reports. Finally, only those who could use computers or mobile phones were included. The estimated models took the random intercept into account so that the study findings could be extrapolated to other Asian American midlife women. Nevertheless, considering that the sample size tended to be small and the data were collected from a selected population (e.g., only those online), the generalization of the study findings is limited.

\section{Conclusions}

In this paper, a culturally tailored physical activity promotion program was pilot-tested on its impact on Asian American midlife women's depressive symptoms through a pilot randomized clinical trial. This study supported the significant effects of the program on the women's active living habits and discrimination stress, but not on other types of physical activity or other outcome variables related to depressive symptoms. Based on the findings, 
suggestions for future research and practice include using a WPAPP to improve Asian American midlife women's depressive symptoms.

First of all, as discussed above, the intervention period of three months might not be long enough to improve the women's physical activity or depressive symptoms. Thus, the intervention period needs to be extended to more than three months. Second, further cultural studies on the women's attitudes toward physical activity and depressive symptoms are needed to understand the exact mechanism through which physical activity promotion could influence Asian American midlife women's depressive symptoms. As discussed above, the women's cultural attitudes might have possibly influenced the impact of the intervention on the outcome variables in a way. Third, more studies on the social media function of a Web-based intervention on the outcome variables related to depressive symptoms are needed to design a Web-based intervention to improve depressive symptom experience of Asian American midlife women. Finally, further studies on the linkage between physical activity and depressive symptoms are warranted to support the underlying assumption of this study (depressive symptoms could be improved by increasing physical activity).

\section{Acknowledgment}

We greatly appreciate the efforts made by Ms. Se Hee Min and Ms. Jia Xue for participant recruitment and data collection.

\section{Funding}

The study was funded by the University Research Foundation Grant and the Dr. Dorothy Mereness Endowed Research Fund at the University of Pennsylvania, and the Chang Gung Medical Research Foundation (ZZRPF3C0011).

\section{References}

Abramson, K., Keefe, B., \& Chou, W.-Y. S. (2015). Communicating about cancer through Facebook: A qualitative analysis of a breast cancer awareness page. Journal of Health Communication, 20, 237-243. doi:10.1080/10810730.2014.927034

Aegisdóttir, S., Gerstein, L. H., \& Çinarbaş, D. C. (2008). Methodological Issues in cross-cultural counseling research equivalence, bias, and translations. The Counseling Psychologist, 36, 188-219. doi:10.1177/0011000007305384

AHRQ. (2014). Patient self-management support programs: An evaluation. Retrieved from http://www.ahrq.gov/research/findings/final-reports/ptmgmt/index.html
Ainsworth, B. E., Sternfeld, B., Richardson, M. T., \& Jackson, K. (2000). Evaluation of the Kaiser Physical Activity Survey in women. Medicine and Science in Sports and Exercise, 32, 1327-1338. doi:10.1097/00005768-200007000-00022

Akaike, H. (1974). A new look at statistical model identification. Ieee Transactions on Automatic Control, 19, 716-723. doi:10.1109/TAC.1974.1100705

Armitage, C. J. (2005). Can the theory of planned behavior predict the maintenance of physical activity? Health Psychology: Official Journal of the Division of Health Psychology, American Psychological Association, 24, 235-245. https://doi.org/10.1037/0278-6133.24.3.235

Bandura, A. (1977). Self-efficacy: Toward a unifying theory of behavioral change. Psychological Review, 84, 191-215. doi:10.1037/0033-295X.84.2.191

Baumeister, R. F., \& Finkel, E. J. (2010). Advanced social psychology: The state of the science. New York, NY: Oxford University Press.

Bell, B. A., Ene, M., Smiley, W., \& Schoeneberger, J. (2013). A multilevel model primer using SAS PROC MIXED. Paper presented at the SAS Global Forum 2013, San Francisco, CA. Retrieved from support.sas.com/resources/papers/proceedings 13/433-2013.pdf

Bernstein, K. S., Park, S.-Y., Shin, J., Cho, S., \& Park, Y. (2011). Acculturation, discrimination and depressive symptoms among Korean immigrants in New York City. Community Mental Health Journal, 47, 24-34. doi:10.1007/s10597-009-9261-0

Chee, W., Kim, S., Chu, T.-L., Tsai, H.-M., Ji, X., Zhang, J., ... Im, E.-O. (2016). Practical issues in developing a culturally tailored physical activity promotion program for Chinese and Korean American midlife women: A pilot study. Journal of Medical Internet Research, 18, e303. https://doi.org/10.2196/jmir.6454

de Snyder, V. N. S. (1987). Factors associated with acculturative stress and depressive symptomatology among married Mexican immigrant women. Psychology of Women Quarterly, 11, 475-488. hdoi:10.1111/j.1471-6402.1987.tb00919.x

Essed, P. (1991). Understanding everyday racism: An Interdisciplinary theory. Atlanta, GA: SAGE Publications.

Gomez, S. L., Kelsey, J. L., Glaser, S. L., Lee, M. M., \& Sidney, S. (2004). Immigration and acculturation in relation to health and health-related risk factors among specific Asian subgroups in a health maintenance organization. American Journal of Public Health, 94, 1977-1984. doi:10.2105/AJPH.94.11.1977

Gwynn, R. C., McQuistion, H. L., McVeigh, K H., Garg, R. K., Frieden, T. R., \& Thorpe, L. E. (2008). Prevalence, diagnosis, and treatment of depression and generalized anxiety disorder in a diverse urban community. Psychiatric Services, 59, 641647. doi:10.1176/appi.ps.59.6.641

Haas, B. (2001). Fatigue, self-efficacy for physical activity, physical activity, and quality of life in women 
with breast cancer (Doctoral dissertation). Retrieved from Virginia Henderson Global Nursing e-Repository.

Hale, L., Troxel, W. M., Kravitz, H. M., Hall, M. H., \& Matthews, K. A. (2014). Acculturation and sleep among a multiethnic sample of women: The Study of Women's Health Across the Nation (SWAN). Sleep, 37, 309-317. doi:10.5665/sleep.3404

Hamer, M., Endrighi, R., \& Poole, L. (2012). Physical activity, stress reduction, and mood: Insight into immunological mechanisms. Methods in Molecular Biology, 934, 89-102. doi:10.1007/978-162703-071-7_5

Han, H.-R., Kim, M. T., \& Weinert, C. (2002). The psychometric evaluation of Korean translation of the Personal Resource Questionnaire 85-Part 2. Nursing Research, 51, 309-316. doi:10.1097/00006199-200209000-00007

Hovey, J. D. (2000). Psychosocial predictors of depression among Central American immigrants. Psychological Reports, 86(3c), 1237-1240.

Im, E.-O., Chang, S. J., Chee, W., \& Chee, E. (2012). Attitudes of women in midlife to web-based interventions for promoting physical activity. Journal of Telemedicine and Telecare, 18, 419-422. doi:10.1258/jtt.2012.120514

Im, E.-O., Chang, S. J., Ko, Y., Chee, W., Stuifbergen, A., \& Walker, L. (2012). A national internet survey on midlife women's attitudes toward physical activity. Nursing Research, 61, 342-352. doi:10.1097/NNR.0b013e31825da85a

Im, E.-O., Chee, W., Lim, H.-J., Liu, Y., \& Kim, H. K. (2008). Midlife women's attitudes toward physical activity. Journal of Obstetric, Gynecologic, and Neonatal Nursing: JOGNN / NAACOG, 37, 203-213. doi:10.1111/j.1552-6909.2008.00219.x

Im, E.-O., \& Choe, M.-A. (2004). Korean women's attitudes toward physical activity. Research in Nursing \& Health, 27, 4-18. doi:10.1002/nur.20000

Im, E.-O., Ko, Y., Chee, E., \& Chee, W. (2015). Cluster analysis of midlife women's sleep-related symptoms: Racial/ethnic differences. Menopause, 22, 1182-1189. doi:10.1097/GME.0000000000000460

Im, E. O., Ko, Y., Chee, E., Chee, W., \& Mao, J. J. (2016). Clusters of midlife women by physical activity and their racial/ethnic differences. Manuscript submitted for publication.

Im, E.-O., Ko, Y., Hwang, H., Chee, W., Stuifbergen, A., Lee, H., \& Chee, E. (2012). Asian American midlife women's attitudes toward physical activity. Journal of Obstetric, Gynecologic, and Neonatal Nursing, 41, 650-658. doi:10.1111/j.15526909.2012.01392.x

Im, E.-O., Shin, H. J., \& Chee, W. (2008). Characteristics of midlife women recruited through internet communities/groups. Computers, Informatics, Nursing, 26, 39-48. doi:10.1097/01.NCN.0000304760.49048.d6
Im, E.-O., Stuifbergen, A. K., \& Walker, L. (2010). A situation-specific theory of Midlife Women's Attitudes Toward Physical Activity (MAPA). Nursing Outlook, 58, 52-58. doi:10.1016/j.outlook.2009.07.001

Im, E.-O., Teng, H., Lee, Y., Kang, Y., Ham, O. K., Chee, E., \& Chee, W. (2014). Physical activities and sleep-related symptoms in 4 major racial/ethnic groups of midlife women. Family \& Community Health, 37, 307-316. doi:10.1097/FCH.0000000000000041

Institute of Medicine (2008). Challenges and successes in reducing health disparities: Workshop summary. Retrieved from http://www.nap.edu/catalog.php?record_id=12154

Jang, Y., Kim, G., \& Chiriboga, D. (2005). Acculturation and manifestation of depressive symptoms among Korean-American older adults. Aging \& Mental Health, 9, 500-507. doi:10.1080/13607860500193021

Jimenez, D. E., Alegría, M., Chen, C.-N., Chan, D., \& Laderman, M. (2010). Prevalence of psychiatric illnesses in older ethnic minority adults. Journal of the American Geriatrics Society, 58, 256-264. doi:10.1111/j.1532-5415.2009.02685.x

Judd, F. K., Hickey, M., \& Bryant, C. (2012). Depression and midlife: Are we overpathologising the menopause? Journal of Affective Disorders, 136, 199211. doi:10.1016/j.jad.2010.12.010

Lagomasino, I. T., Dwight-Johnson, M., Miranda, J., Zhang, L., Liao, D., Duan, N., \& Wells, K. B. (2005). Disparities in depression treatment for Latinos and site of care. Psychiatric Services, 56, 1517-1523. doi:10.1176/appi.ps.56.12.1517

Lavallee, L., \& Flint, F. (1996). The relationship of stress, competitive anxiety, mood state, and social support to athletic injury. Journal of Athletic Training, 31, 296-299.

Lee, L.-L., Arthur, A., \& Avis, M. (2008). Using self-efficacy theory to develop interventions that help older people overcome psychological barriers to physical activity: A discussion paper. International Journal of Nursing Studies, 45, 16901699. doi:10.1016/j.ijnurstu.2008.02.012

Masuda, M., \& Holmes, T. H. (1978). Life events: Perceptions and frequencies. Psychosomatic Medicine, 40, 236-261. doi:10.1097/00006842-19780500000006

McDonnell, D. D., Lee, H.-J., Kazinets, G., \& Moskowitz, J. M. (2010). Online recruitment of targeted populations: Lessons learned from a smoking cessation study among Korean Americans. Social Marketing Quarterly, 16(3), 2-22. doi:10.1080/15245004.2010.500441

Noh, S., Avison, W. R., \& Kaspar, V. (1992). Depressive symptoms among Korean immigrants: Assessment of a translation of the Center for Epidemiologic Studies-Depression Scale. Psychological Assessment, 4, 84-91. doi:10.1037/10403590.4.1.84 
Park, S.-Y., \& Bernstein, K. S. (2008). Depression and Korean American immigrants. Archives of Psychiatric Nursing, 22, 12-19. doi:10.1016/j.apnu.2007.06.011

Parry, B. L. (2010). Optimal management of perimenopausal depression. International Journal of Women's Health, 2, 143-151. doi:10.2147/IJWH.S7155

Polit, D. F., \& Beck, C. T. (2014). Essentials of nursing research: Methods, appraisal, and utilization. Philadelphia, PA: Lippincott Williams \& Wilkins.

Rydell, S. A., French, S. A., Fulkerson, J. A., NeumarkSztainer, D., Gerlach, A. F., Story, M., \& Christopherson, K. K. (2005). Use of a Web-based component of a nutrition and physical activity behavioral intervention with Girl Scouts. Journal of the American Dietetic Association, 105, 14471450. doi:10.1016/j.jada.2005.06.027

Shin, H. S., Han, H.-R., \& Kim, M. T. (2007). Predictors of psychological well-being amongst Korean immigrants to the United States: A structured interview survey. International Journal of Nursing Studies, 44, 415-426. doi:10.1016/j.ijnurstu.2006.04.007

Sin, M.-K., Jordan, P., \& Park, J. (2011). Perceptions of depression in korean american immigrants. Issues in Mental Health Nursing, 32, 177-183. doi:10.3109/01612840.2010.536611

Spector, R. E. (2012). Cultural diversity in health and illness (8th ed.). Boston, MA: Pearson.

Spitzer, R. L., Kroenke, K., Williams, J. B. W., \& the Patient Health Questionnaire Primary Care Study Group (1999). Validation and utility of a self-report version of PRIME-MD: The PHQ primary care study. Journal of the American Medical Association, 282, 1737-1744. doi:10.1001/jama.282.18.1737

Stuifbergen, A. K., \& Becker, H. A. (1994). Predictors of health-promoting lifestyles in persons with disabilities. Research in Nursing \& Health, 17, 3-13.

Suinn, R. M., Ahuna, C., \& Khoom, G. (1992). The SuinnLew Asian Self-Identity Acculturation Scale: Concurrent and factorial validation. Educational and Psychological Measurement, 52, 1041-1046. doi:10.1177/0013164492052004028
Torres, E. R., Sampselle, C. M., Ronis, D. L., Neighbors, H. W., \& Gretebeck, K. A. (2013). Leisure-time physical activity in relation to depressive symptoms in African-Americans: Results from the National Survey of American Life. Preventive Medicine, 56, 410-412. doi:10.1016/j.ypmed.2013.02.013

Trivedi, M. H., Rush, A. J., Wisniewski, S. R., Nierenberg, A. A., Warden, D., Ritz, L., ... STAR*D Study Team. (2006). Evaluation of outcomes with citalopram for depression -using measurement-based care in STAR*D: Implications for clinical practice. The American Journal of Psychiatry, 163, 28-40. doi:10.1176/appi.ajp.163.1.28

Turner, R. J., \& Avison, W. R. (2003). Status variations in stress exposure: Implications for the interpretation of research on race, socioeconomic status, and gender. Journal of Health and Social Behavior, 44, 488-505. doi:10.2307/1519795

U.S. Census Bureau. (2011). Asian/Pacific American heritage month: May 2011. Retrieved from https://www.census.gov/newsroom/releases/archives/facts_for_features_special_editions/cb11ff06.html

Weinert, C. (2003). Measuring social support: PRQ2000. In O. Strickland \& C. DiIorio (Eds.), Measurement of nursing outcomes: Vol. 3. Self care and coping (pp. 161-172). New York, NY: Springer.

Weissman, M. M., Leaf, P. J., Holzer, C. E., III, Myers, J. K., \& Tischler, G. L. (1984). The epidemiology of depression. An update on sex differences in rates. Journal of Affective Disorders, 7, 179-188. doi:10.1016/0165-0327(84)90039-9

Wilbur, J., Buchholz, S. W., Ingram, D. M., Braun, L. T., Johnson, T. J., Fogg, L., ... McDevitt, J. (2013). Effectiveness, efficiency, duration, and costs of recruiting for an African American women's lifestyle physical activity program. Research in Nursing \& Health, 36, 487-499. doi:10.1002/nur.21550

Wise, L. A., Adams-Campbell, L. L., Palmer, J. R., \& Rosenberg, L. (2006). Leisure time physical activity in relation to depressive symptoms in the Black Women's Health Study. Annals of Behavioral Medicine, 32, 68-76. doi:10.1207/s15324796abm3201_8 